\title{
The gridGraphics Package
}

by Paul Murrell

Abstract The gridGraphics package provides a function, grid. echo(), that can be used to convert a plot drawn with the graphics package to a visually identical plot drawn using grid. This conversion provides access to a variety of grid tools for making customisations and additions to the plot that are not possible with the graphics package.

\section{Introduction}

The core graphics system in $\mathrm{R}$ is divided into two main branches, one based on the graphics package and one based on the grid package, with many other packages building on top of one or other of these graphics systems (see Figure 1).

The graphics package is older and provides an emulation of the original GRZ graphics system from S (Becker and Chambers, 1984). The newer grid package, although its performance is actually slower, provides greater flexibility and additional features compared to the graphics package. In particular, a plot drawn with grid can be manipulated and edited in many more ways than a plot drawn with the graphics package.

This article describes a new package, called gridGraphics, that allows a plot drawn with graphics to be converted into an identical plot drawn with grid, thereby allowing the plot to be manipulated using all of the tools available in grid.

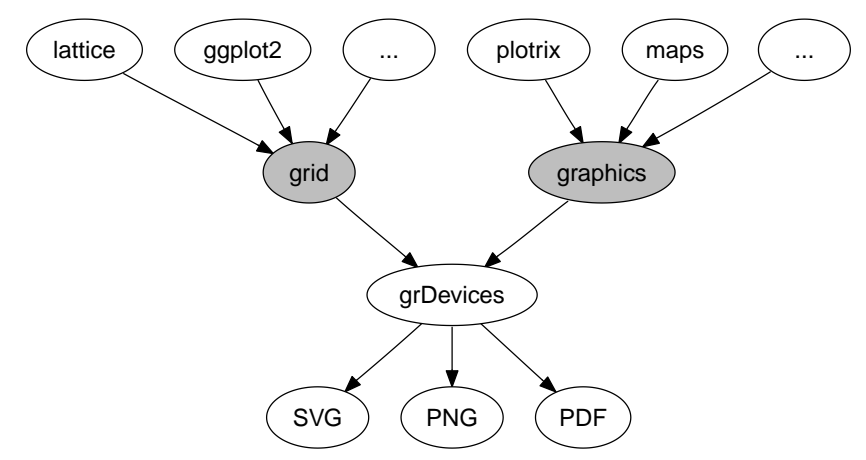

Figure 1: The structure of the core graphics system in R. The lattice package (Sarkar, 2008), the ggplot2 package (Wickham, 2009) and many others are built on top of the grid package; the plotrix package (Lemon, 2006), the maps package (Brownrigg, 2013) and many others are built on top of the graphics package.

\section{The grid.echo() function}

The gridGraphics package provides a single main function called grid.echo(). By default, this function takes whatever has been drawn by the graphics package on the current graphics device and redraws it using grid. The following code provides a simple demonstration. We first draw a scatterplot using plot() from the graphics package, then we call grid.echo() to replicate the plot with grid. Figure 2 shows that the original plot and the replicated plot are identical.

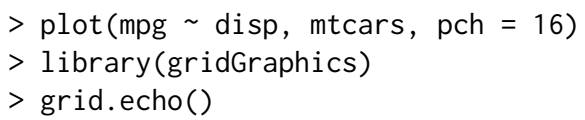

The following sections will attempt to demonstrate why, although the plots appear identical to the eye, there are important advantages that arise from using grid to do the drawing.

\section{Manipulating grobs}

One advantage of drawing the plot with grid is that there is an object, a grid grob, recorded for each separate component of the plot that we have drawn. We can see that list of grobs with a call 


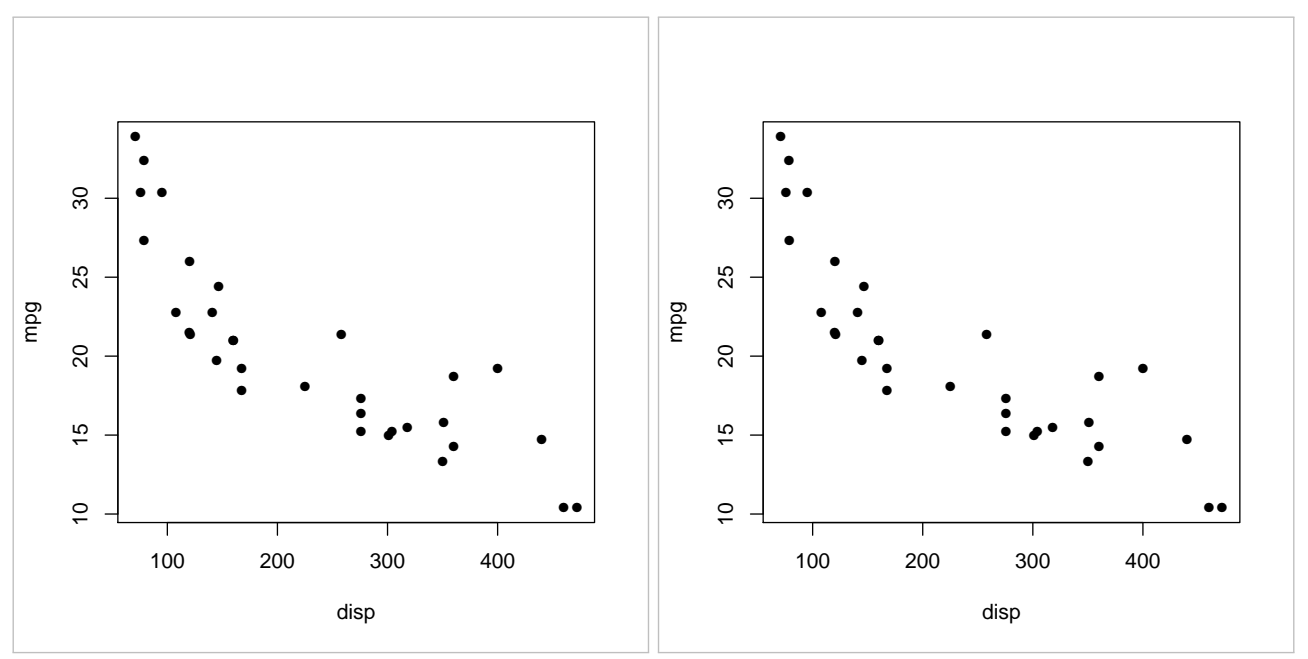

Figure 2: On the left, a scatterplot drawn with the graphics package and, on the right, the result of grid.echo(), which produces the same scatterplot using the grid package.

to the grid.1s() function, as shown below. There is a grob called graphics-plot-1-points-1 that represents the data symbols in the plot, there is a grob called graphics-plot-1-xlab-1 that represents the $\mathrm{x}$-axis label, and so on.

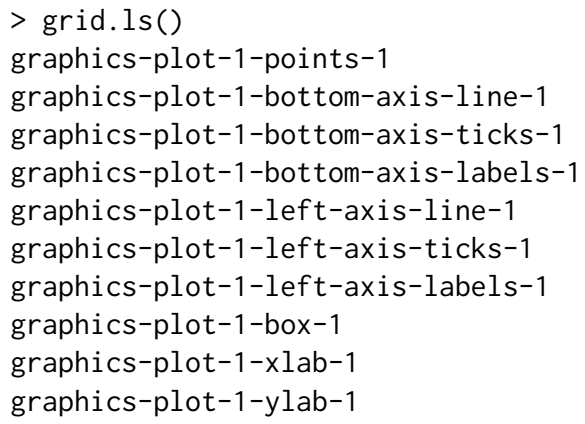

The grid package provides several functions to manipulate these grobs. For example, the code below uses the grid.edit() function to rotate the tick labels on the x-axis of the plot to 45 degrees (and turns them red so that the change is easy to spot; see Figure 3). This is a simple example of a customisation that is impossible or very difficult in the graphics package, but is quite straightforward once the plot has been converted to grid.

$>$ grid.edit("graphics-plot-1-bottom-axis-labels-1", rot = 45,

$+\quad \operatorname{gp}=\operatorname{gpar}(\mathrm{col}=$ "red"))

To provide a more sophisticated example, consider the conditioning plot produced by the following code with a call to the coplot () function from the graphics package (see the left-hand plot in Figure 4).

$>\operatorname{coplot}($ lat $\sim$ long I depth, quakes, pch $=16$, cex $=.5$,
$+\quad$ given.values $=\operatorname{rbind}(c(0,400), c(300,700)))$

This is an example of a much more complex plot with many different components. Functions that produce this sort of complex plot can struggle to provide arguments to fine tune all possible elements of the plot. For example, suppose that we want to modify the "conditioning panel" at the top of the plot so that the background is a solid colour and the bars are filled in white. This specific task is probably not a common one for most people, but the point of this example is to represent a class of problems where a small detail within a complex plot needs to be modified.

The coplot() function does have an argument bar.bg to control the fill colour for the bars, as demonstrated in the code below (see the right-hand plot in Figure 4). However, there is no argument that allows us to control the background colour for the panel behind the bars.

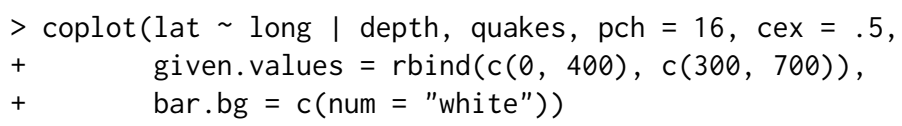




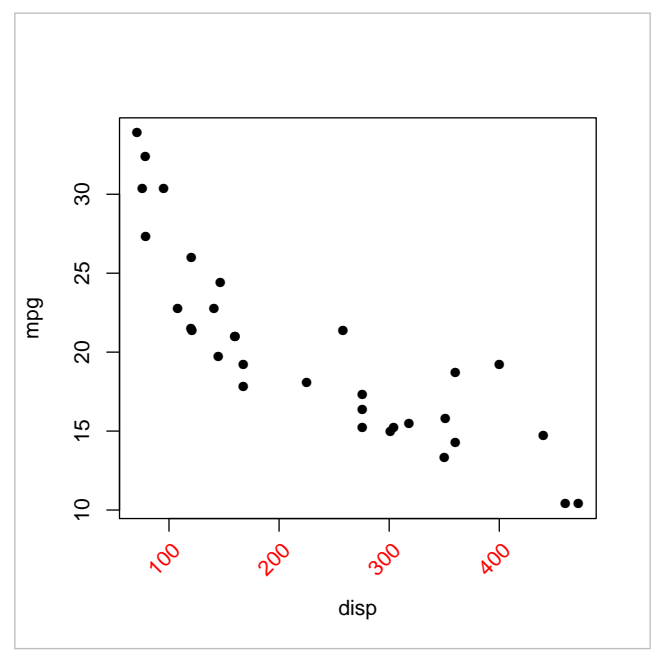

Figure 3: A scatterplot that was drawn using $\operatorname{plot}($ ) from graphics, then redrawn using grid via the grid.echo() function, then edited using grid.edit().

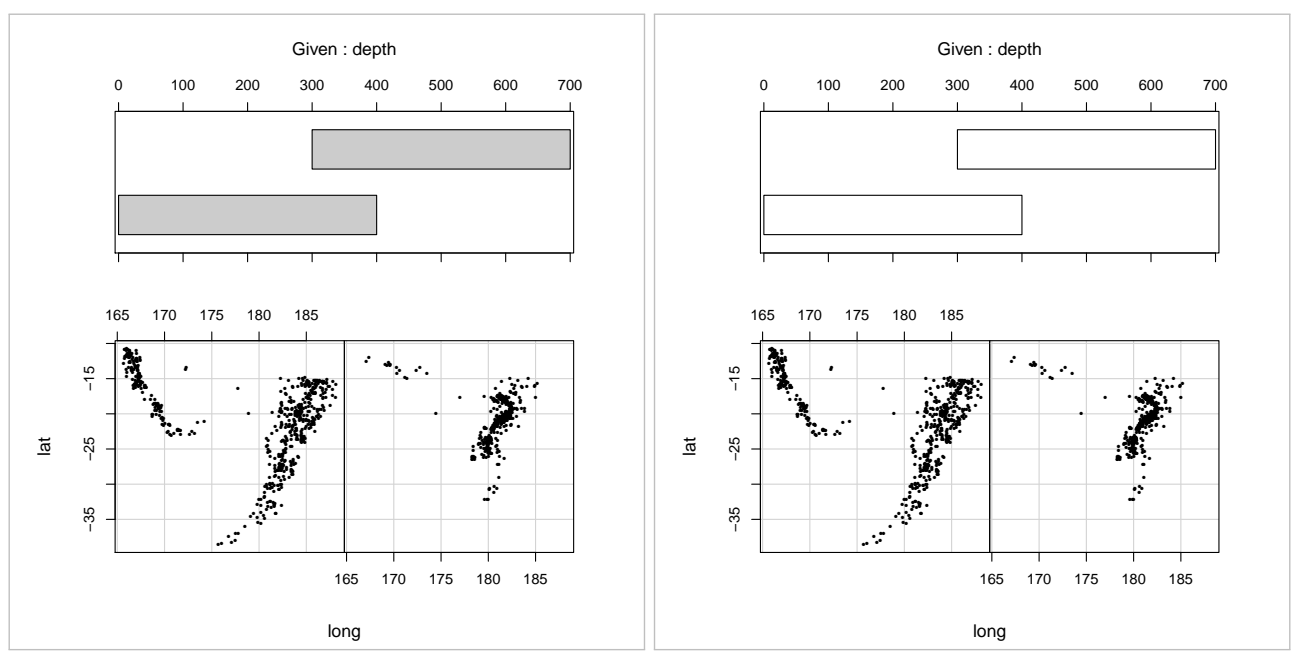

Figure 4: A conditioning plot produced by the $\operatorname{coplot}($ ) function from the graphics package. The right-hand version of the plot demonstrates the use of the bar . bg argument to customise the fill colour of the bars in the conditioning panel at the top of the plot.

If we replicate this plot using grid, we have more tools available to be able to manipulate the plot. The grid.echo() function can replicate this plot and gives an identical result to that shown in Figure 4.

The call to grid.echo() shown below also demonstrates the use of the prefix argument, which can be used to control the naming of the grobs that grid.echo() draws. The grobs created by this call to grid.echo() all have names that start with " $\mathrm{cp}$ " rather than the default "graphics" prefix.

$>$ grid.echo(prefix $=" c p ")$

Once this conversion has taken place, we now have grid grobs that represent all components of the plot. In particular, there is a grob called "cp-plot-4-box-1" that draws the border around the conditioning panel. We can edit that grob to give it a fill colour (see the left-hand plot in Figure 5).

$>$ grid.edit("cp-plot-4-box-1", gp = $\operatorname{gpar}(f i l l=$ "red"))

This provides another demonstration that converting a plot to grid provides access to all of the components of the plot, which allows fine control over details of the plot that cannot be controlled via the arguments to the original high-level function that created the plot.

In this particular example, we have also created a new problem, because the border on the conditioning plot is drawn after the bars, so the bars are now obscured. Fortunately, we can fix this as well with further tools that grid provides for manipulating grobs. 
In the following code, we call the grid.grab() function to create a single grob (a gTree) that contains all of the other grobs on the page. We then call the grid. reorder() function to change the order of the grobs within the gTree. The code specifies that the border grob will be drawn first (behind all other components in the plot). Finally, we redraw the reordered plot with the grid.draw() function to get the final result that we were after (see the right-hand plot in Figure 5).
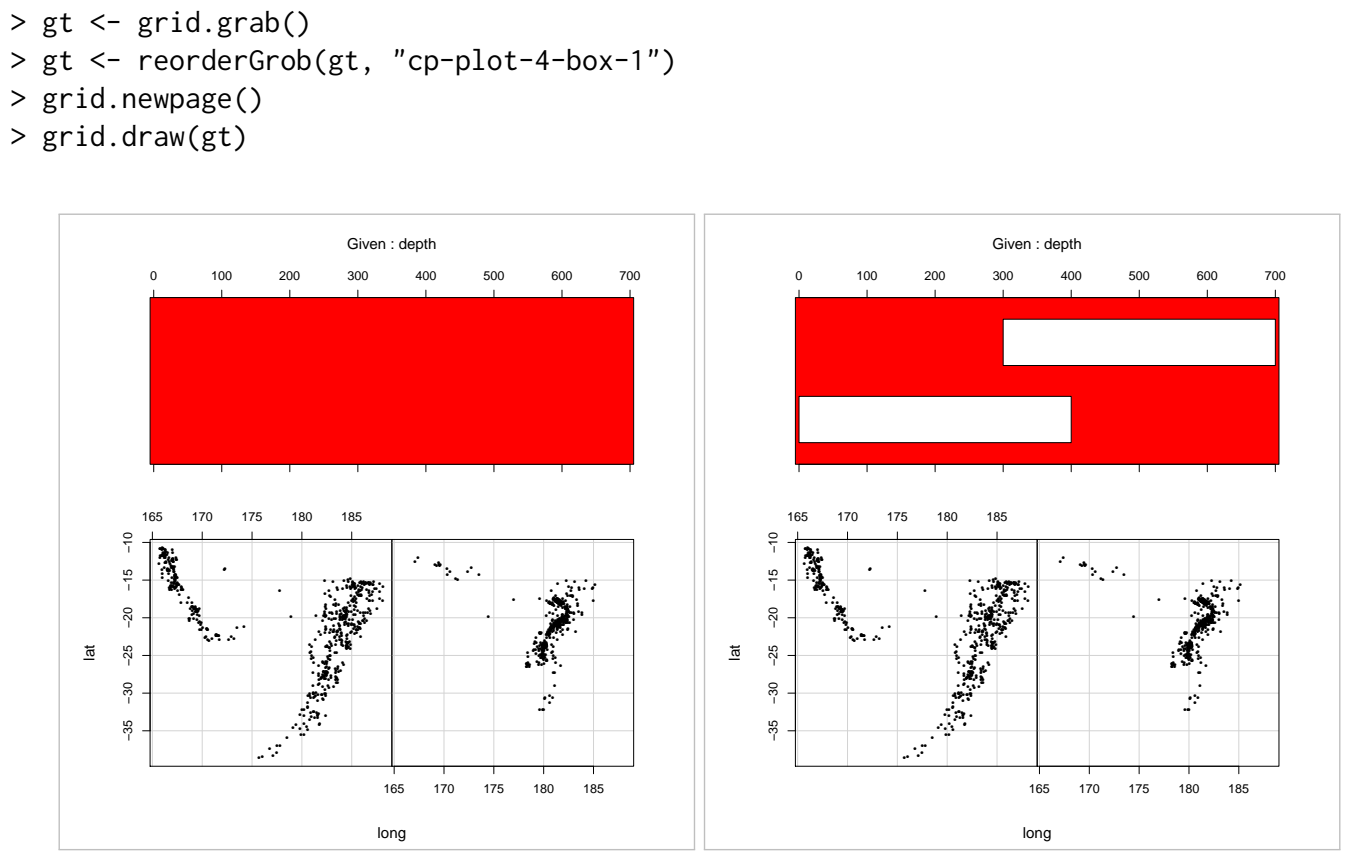

Figure 5: On the left, the plot from Figure 4 after conversion with grid.echo(), followed by editing to fill the rectangle that draws a border around the top conditioning panel (in red so that the change is visible). On the right, the edited plot has been reordered so that the border around the conditioning panel is drawn first (behind everything else).

This access to individual components of a plot and the ability to manipulate those components is one benefit of converting a graphics plot to grid.

\section{Making use of viewports}

Another advantage of using grid is that we can make use of viewports. Viewports are similar to the different plotting regions that the graphics package uses for drawing (see Figure 6), but in a grid plot there can be an unlimited number of viewports and all viewports are accessible at any time. In the graphics package there is only the current plot region, figure margins, and outer margins to work with.

Figure 7 shows a diagram of the hierarchy of viewports that grid.echo() created when we replicated the simple scatterplot in Figure 2. This shows that gridGraphics produces quite a lot of viewports (even for a simple plot), but there is a coherent structure to the viewports, so the complexity can be navigated without too much difficulty.

In general, the names of the viewports reflect the plot regions that they mimic in the original plot. At the top of the hierarchy of viewports is a viewport called ROOT, which represents the entire page (this viewport is always present). Below that is a viewport called graphics-root and that represents the area of the page that grid. echo() has drawn into (by default, also the whole page). The next viewport down is called graphics-inner and this represents the region that is the whole page minus the outer margins. Below that are two viewports, graphics-figure-1 and graphics-figure-1-clip, both of which correspond to the figure region (the grey area in Figure 6). There are two viewports because one has clipping turned on, so that drawing within that region cannot extend beyond the boundaries of the region, and one has clipping turned off. Below each of the figure region viewports are one or more viewports representing the plot region, called either graphics-plot-1 or graphics-plot-1-clip. Again, the difference between these two plot viewports is whether clipping is on or off. By having the graphics-plot-1 viewport beneath both figure viewports, we can represent all possible values of the par ("xpd") settings: clipping to the figure region, or clipping to the plot region, or no clipping at all. The bottom layer of viewports represent the plot region again, but this time with a viewport that has 


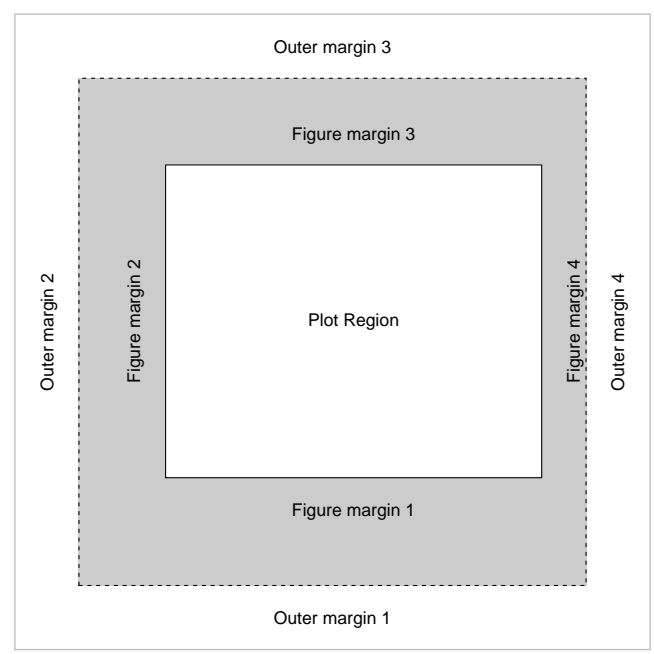

Figure 6: The plotting regions involved in the drawing of a graphics plot.

scales to represent the plot axes. The reason for this additional layer is so that we can reproduce plots that make use of more than one set of axes (e.g., two different y-axis scales).

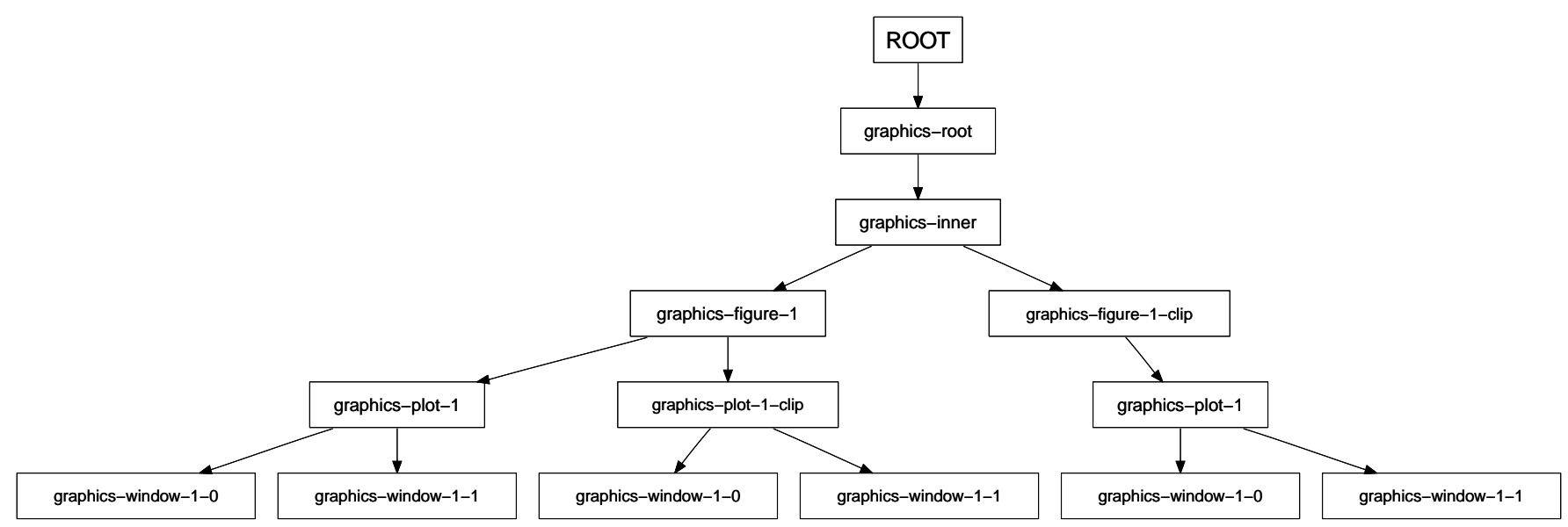

Figure 7: A diagram of the viewports that were created by the grid. echo() function when it drew the plot in Figure 2.

The upside to having so many viewports is that the grid package provides functions to navigate between viewports. So we can have a lot of viewports on the page at once, but switch between them if we want to add drawing within different viewports. As an example, the following code uses the downViewport() function to revisit the plot region viewport that was created by grid.echo() and draws a red rectangle around the border (see Figure 8). The upViewport () function is then used to take us back to the whole page (the ROOT viewport).

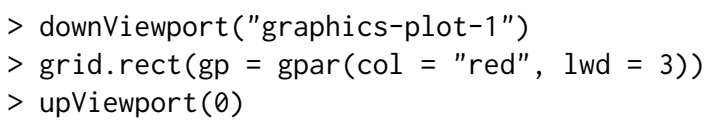

Once again, a more sophisticated demonstration can be provided if we consider the more complex conditioning plot from Figure 4. Another limitation of the coplot () function, because it is based on the graphics package, is that there is no way to add further drawing to the conditioning panel at the top of the plot.

This plot has several different panels so the replication created by grid.echo() generates many different viewports, including viewports used to draw the conditioning panel at the top of the plot. 


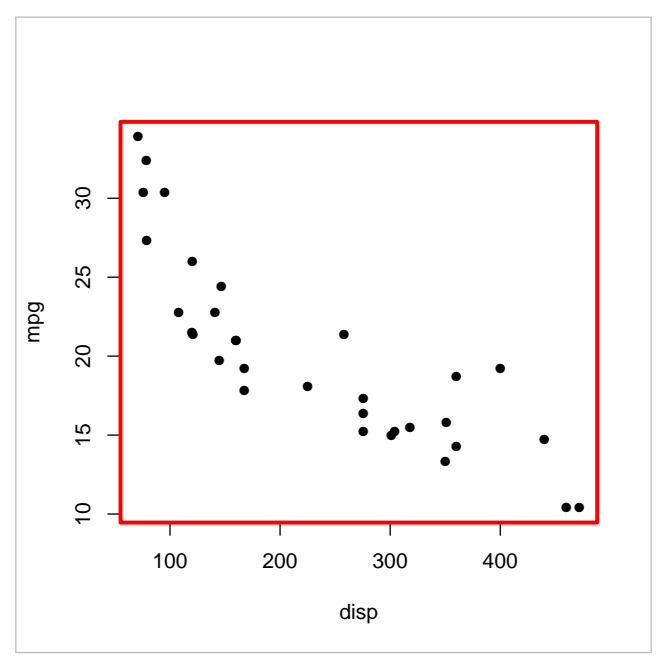

Figure 8: The echoed plot from Figure 2 with a rectangle added by revisiting the viewport that corresponds to the plot region.

With grid, all of these viewports can be revisited after the plot has been drawn. In the following code, we revisit the viewport used to draw the conditioning panel and draw some grid lines in it.

Those new lines will be drawn on top of everything else, so additional manipulations, similar to the reordering performed for Figure 5, can also be carried out to push the segments behind everything else (code not shown). The result is shown in Figure 9.
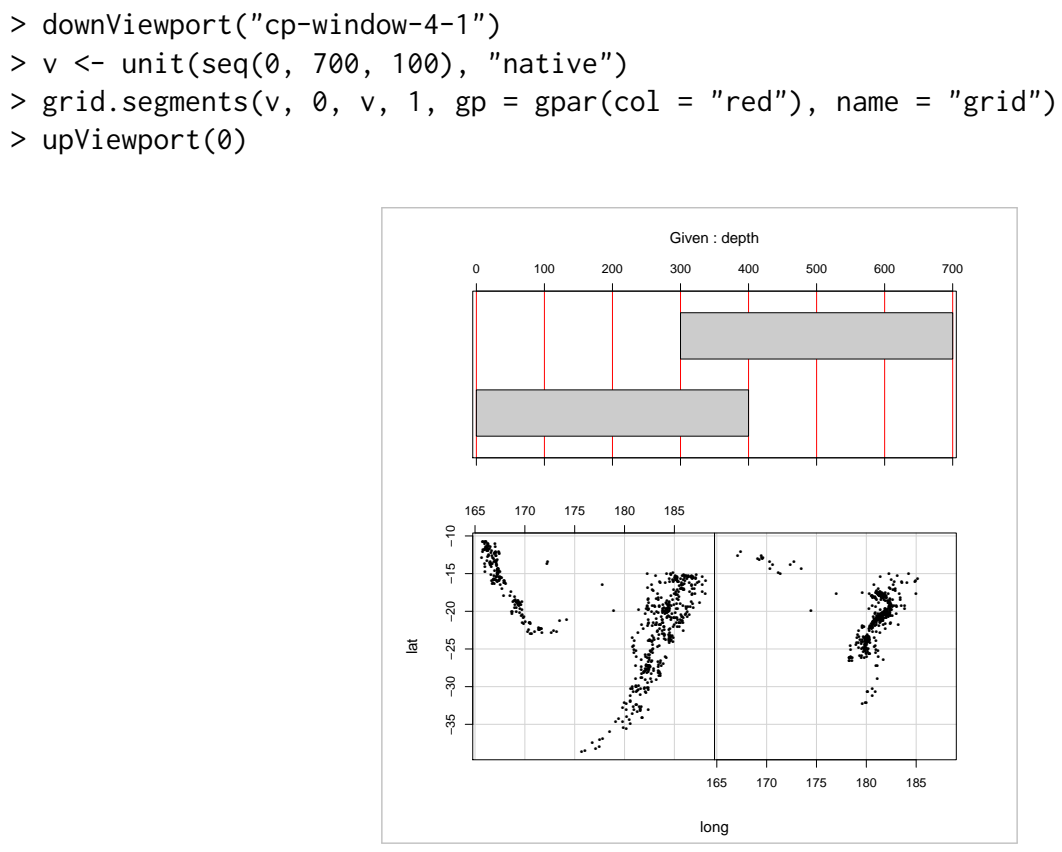

Figure 9: The conditioning plot from Figure 4 with a reference grid added (in red so that the change is visible) to the conditioning panel at the top of the plot.

Another limitation of the original coplot() function is that it insists on occupying the entire page. Another advantage of working with grid grobs and viewports is that they can be nested within each other to any level. This means that once the output from coplot() has been replicated as grid output, it can be drawn within a grid viewport and combined on a page with other plots.

The following code creates a grid viewport occupying the bottom $70 \%$ of the page and then replicates the conditioning plot only using that part of the page. This code demonstrates another way to call the grid.echo() function. Rather than calling the graphics function coplot () to draw a plot and then calling grid.echo() to replicate it, we can define a function (with no arguments) that draws the plot and then provide that function as the first argument to grid.echo(). This way the plot is only drawn once, using grid. We also specify newpage = FALSE in the call to grid.echo() so that it just draws in the current viewport rather than starting a new page. 


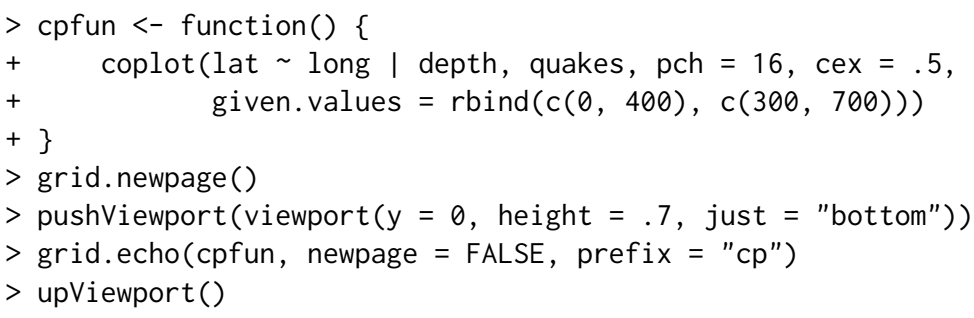

The next piece of code draws a ggplot2 histogram in the top third of the page, so not only do we have a conditioning plot combined with another plot on the same page (something that was not at all possible with the original graphics-based conditioning plot), but we have a mixture of graphics-based and grid-based output on the same page (see Figure 10).
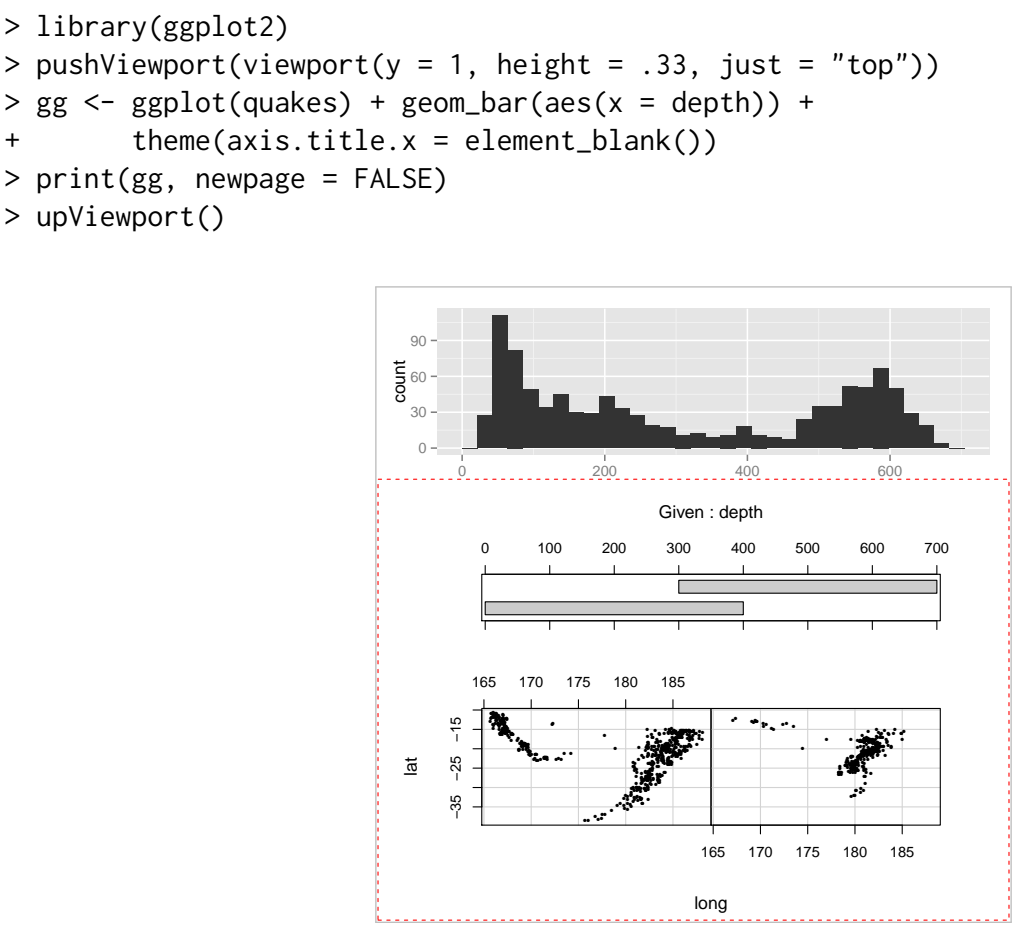

Figure 10: The conditioning plot from Figure 4 combined with a ggplot2 histogram on the same page. A dashed red box has been drawn around the region that is occupied by the conditioning plot, to emphasise the fact that the conditioning plot does not occupy the entire page.

This sort of result-grid-based plots combined with graphics-based plots on the same page-can also be achieved using the gridBase package (Murrell, 2012). However, gridBase only allows plots from the two packages to coexist side-by-side on the same page; it does not provide any of the benefits of grid for graphics-based plots.

\section{Exporting to SVG}

Another benefit that we get from converting a graphics plot to grid is that the converted plot can then be exported to SVG via the gridSVG package (Murrell and Potter, 2014). This means that we gain the potential to add hyperlinks to the plot, animate components of the plot, add advanced SVG features to the plot, and add interactivity (possibly via JavaScript code).

As a simple example, the following code draws the scatterplot from Figure 2 with plot() from the graphics package, converts the plot to grid with grid.echo(), and then adds tooltips to each data symbol and exports the plot to SVG with the functions grid.garnish() and grid.export() from the gridSVG package. The SVG plot, as viewed in a browser, is shown in Figure 11.

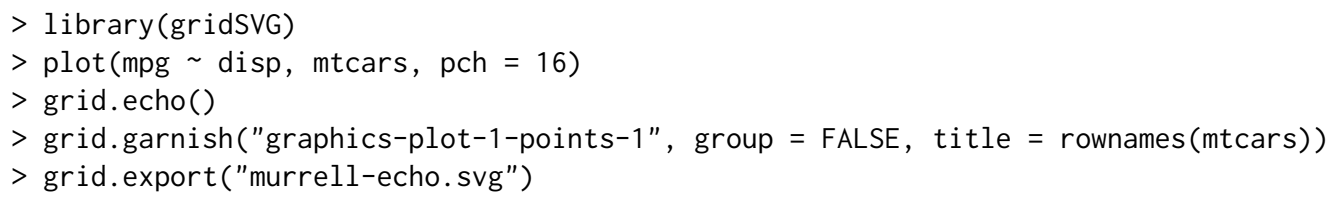




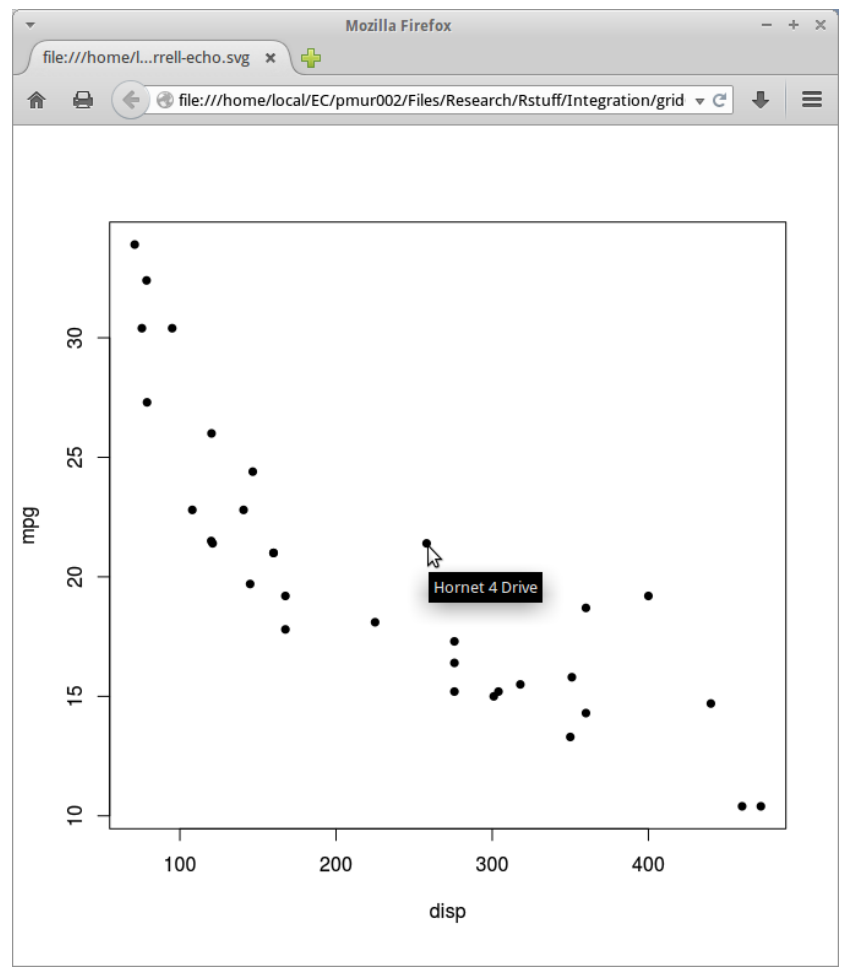

Figure 11: The scatterplot plot from Figure 2, after conversion to grid using grid.echo(), exported to SVG, with tooltips added, using functions from the gridSVG package. The tooltips in this example may only work with Firefox.

A graphics plot that is converted by gridGraphics and then exported by gridSVG also has potential benefits for accessibility because, for example, text labels are exported as <text> elements, which can be recognised by screen reading software. This is not the case with the standard $\operatorname{svg}()$ graphics device, which exports text as $<$ path $>$ elements.

\section{Naming schemes}

Something that has been ignored in the examples so far is the fact that all manipulations of grid grobs, as well as navigation between grid viewports, rely on being able to identify, by name, which grob we want to manipulate or which viewport we want to visit. This section describes the naming scheme that the gridGraphics package uses to assign names to the grobs and viewports that it creates.

The names of grobs have the following basic pattern:

$<$ prefix $>-$ plot $-<\mathrm{i}>-<$ label $>-<j>$

where $<$ prefix $>$ is graphics by default, but can be specified in the call to grid.echo(), $<i>$ reflects which plot the grob was drawn in (because there can be more than one plot region on the page), $<$ label $>$ reflects what sort of shape was drawn, and $\langle j\rangle$ is a numeric index that automatically increments when more than one of the same shape is drawn within the same plot. The full set of possible shape labels is given in Table 1.

The names of viewports have one of the following basic patterns:

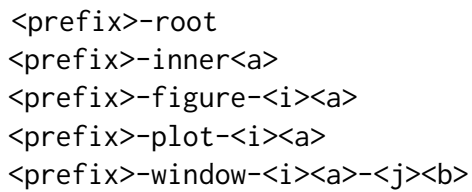

where $<$ prefix $>$ is the same as for grob names. There is only ever one root viewport, which is parent to usually one inner viewport. Both plot and window viewports occupy the same region, but window viewports represent the axis scales. The $<i>$ part of the name is a numeric index that is automatically incremented, each time that plot. new () is called. The $\langle j\rangle$ part is similar, but increments each time 


\begin{tabular}{|c|c|c|}
\hline Function & Description & $<$ label $>$ \\
\hline plotXY() & Points, lines, etc. through data & $\begin{array}{l}\text { points } \\
\text { lines } \\
\text { step } \\
\text { Step } \\
\text { spike } \\
\text { brokenline }\end{array}$ \\
\hline $\operatorname{text}()$ & Text in plot region & text \\
\hline title() & Plot title, sub-title, and axis labels & $\begin{array}{l}\text { main } \\
\text { sub } \\
\text { xlab } \\
\text { ylab }\end{array}$ \\
\hline $\operatorname{axis}()$ & $\begin{array}{l}\text { Axes, including tick marks and } \\
\text { labels }\end{array}$ & $\begin{array}{l}\text { bottom-axis-line } \\
\text { bottom-axis-ticks } \\
\text { bottom-axis-labels } \\
\text { (ditto left-, top-, and right-) }\end{array}$ \\
\hline mtext() & Text in margins & $\begin{array}{l}\text { mtext-bottom } \\
\text { mtext-left } \\
\text { mtext-top } \\
\text { mtext-right } \\
\text { mtext-<side>-outer }\end{array}$ \\
\hline $\operatorname{box}()$ & Border rectangles for plot regions & $\begin{array}{l}\text { box } \\
\text { box-figure } \\
\text { box-inner } \\
\text { box-outer }\end{array}$ \\
\hline segments() & Straight line segments & segments \\
\hline $\operatorname{arrows}()$ & Segments with arrow heads & arrows \\
\hline abline() & $\begin{array}{l}\text { A straight line parameterised by } \\
\text { slope and intercept, or horizontal } \\
\text { or vertical constant }\end{array}$ & $\begin{array}{l}\text { abline-ab } \\
\text { abline-h } \\
\text { abline-v }\end{array}$ \\
\hline $\operatorname{rect}()$ & Rectangles & rect \\
\hline polygon() & Polygons & polygon \\
\hline path() & General path & path \\
\hline raster Image() & Raster image & raster \\
\hline xspline() & X-Splines & xspline \\
\hline $\operatorname{clip}()$ & Clipping region rectangle & clip \\
\hline contour () & Contour lines & contour $-<i>$ \\
\hline image() & Filled rectangles & image-rect \\
\hline symbols() & High-dimensional data symbols & $\begin{array}{l}\text { symbols-circle } \\
\text { symbols-square } \\
\text { symbols-rect } \\
\text { symbols-star } \\
\text { symbols-thermo-box } \\
\text { symbols-thermo-fill } \\
\text { symbols-thermo-whisker-right } \\
\text { symbols-thermo-whisker-left } \\
\text { symbols-boxplot-box } \\
\text { symbols-boxplot-lower-whisker } \\
\text { symbols-boxplot-upper-whisker } \\
\text { symbols-boxplot-median }\end{array}$ \\
\hline
\end{tabular}

Table 1: The labels used for different shapes (grobs) that can be created by grid.echo(). The "Function" column gives the name of the graphics function that produces the original shapes, the "Description" column describes what sort of shape is drawn, and the "<label $>$ " column gives the names used for the grid grobs that the shapes are converted into. 
that plot.window() is called. The <a> part of the name only occurs when par () is used to modify graphical parameters that affect the location of plot regions, for example, to modify the plot region via par ("pin"). The $<b>$ part is similar, but occurs when par ("usr") is used to modify the axis scales.

For complex plots, like the conditioning plot in Figure 4, there can be a large number of grobs and viewports. To help with exploring the potentially large number of grobs and viewports, the grid package provides functions grid.ls(), showGrob(), and showViewport(), and the gridDebug package (Murrell and Ly, 2012) provides further tools.

\section{Testing}

The gridGraphics package is known to produce identical results for all examples in the graphics package help pages, plus the results of demo ("graphics") (subject to the caveats described in the next section). However, it is still possible that there are some combinations of arguments in the low-level graphics functions that will not be emulated correctly by grid. echo().

To test whether a graphics-based plot is reproduced correctly by grid.echo(), the gridGraphics package provides a plotdiff() function. This takes an expression as its first argument, which is assumed to be one or more calls to graphics functions. The plotdiff() function creates PDF and PNG files from evaluating the expression and from converting the result with grid.echo(), plus a PNG file showing differences between the graphics original and the grid copy, if there are any (a total of either four or five files). ${ }^{1}$ A plotdiffResult () function is also provided to summarise the results of multiple calls to plotdiff().

\section{Limitations}

There are a few graphics functions that grid.echo() cannot currently replicate: persp() for drawing 3-dimensional surfaces, filled. contour() for drawing a filled contour plot, and recordGraphics(), which allows delayed evaluation of drawing code.

For some other functions, there are a few details that do not reproduce exactly. For example, grid.echo() cannot reproduce text labels on contours drawn by contour() and grid.echo() will sometimes eliminate fewer axis tick labels than the axis() function.

More generally, grid.echo() cannot cope with code that opens or closes graphics devices or changes the current graphics device, it can only replicate a single page of plots, and if there is already a mixture of grid and graphics plots on a page, grid.echo() will only replicate the graphics plots.

An important situation where the above limitations will be encountered is within the RStudio IDE (RStudio, 2014). Plots drawn in the RStudio "Plots" pane will not reproduce well with grid.echo(). However, using a standard R graphics device with RStudio should still work.

Finally, the output from grid.echo() is only valid at the size it is first drawn. Subsequently resizing the graphics window or copying to another graphics device is likely to produce a distorted result.

\section{Conclusion}

The gridGraphics package provides a bridge between the graphics and grid packages (see Figure 12). The grid.echo() function converts a plot drawn using the graphics package into exactly the same result drawn using grid. In effect, the gridGraphics package provides an automated way to create a grid-based version of almost any plotting function that is based on the graphics package.

The benefit of converting to grid is that grid provides tools for making customisations and additions to a plot that are not possible with the graphics package:

- To allow customisation of fine details that are not accessible via the graphics package (e.g., Figures 3 and 5).

- To add extra drawing to a region of the plot that is inaccessible via the graphics package (e.g., Figure 9).

- To combine graphics-based plots with grid-based plots (e.g., Figure 10).

\footnotetext{
${ }^{1}$ On R versions prior to 3.2.0, and on systems where ImageMagick (ImageMagick Studio LLC, 2014) is not available, only the PDF files are created; there is no conversion or comparison. Prior to R 3.2.0, there will be some (typically very small) differences between some plots because of a bug in grid.
} 
- To export a graphics-based plot to SVG with the gridSVG package (e.g., Figure 11).

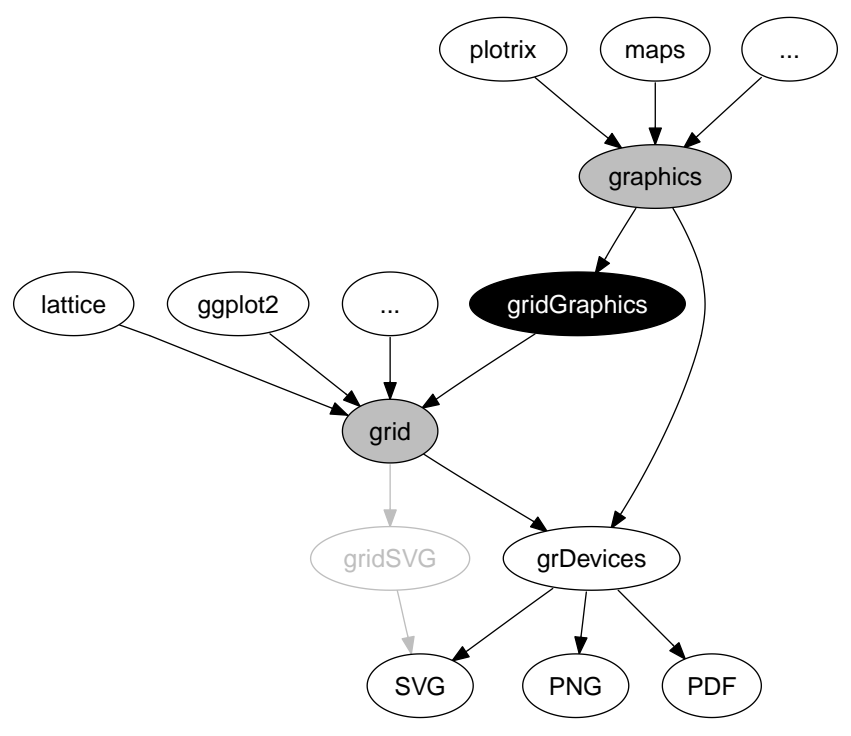

Figure 12: How the gridGraphics package fits into the structure of the core graphics system in $\mathrm{R}$. The gridSVG package is also shown as an alternative route from grid to SVG output.

\section{Availability}

The gridGraphics package is available from CRAN, with ongoing development occurring on github. ${ }^{2}$ Supporting material for this article, including a live version of Figure 11, is available from the following web site:

https://www.stat.auckland.ac.nz/ paul/Reports/gridGraphics/

\section{Acknowledgements}

Thanks to the anonymous reviewers who made several useful suggestions that improved this manuscript.

\section{Bibliography}

R. A. Becker and J. M. Chambers. S: An Interactive Environment for Data Analysis and Graphics. Wadsworth, Belmont, CA, 1984. [p151]

R. Brownrigg. maps: Draw Geographical Maps, 2013. URL http://CRAN. R-project . org/package=maps. $\mathrm{R}$ package version 2.3-2. [p151]

ImageMagick Studio LLC. ImageMagick, 2014. URL http://www. imagemagick. org/. [p160]

J. Lemon. plotrix: A package in the red light district of R. R News, 6(4):8-12, 2006. [p151]

P. Murrell. gridBase: Integration of base and grid Graphics, 2012. URL http://CRAN. R-project.org/ package=gridBase. R package version 0.4-6. [p157]

P. Murrell and V. Ly. Debugging grid graphics. The R Journal, 4(2):19-27, Dec. 2012. URL http: //journal.r-project.org/archive/2012-2/RJournal_2012-2_Murrel1+Ly.pdf. [p160]

P. Murrell and S. Potter. The gridSVG package. The R Journal, 6(1):133-143, June 2014. URL http: //journal.r-project.org/archive/2014-1/murrell-potter.pdf. [p157]

RStudio. RStudio: Integrated Development Environment for R, 2014. URL http://www. rstudio.org/. [p129, 160]

\footnotetext{
${ }^{2}$ https://github.com/pmur002/gridgraphics
} 
D. Sarkar. lattice: Multivariate Data Visualization with R. Springer, New York, 2008. URL http: //lmdvr.r-forge.r-project.org. [p151]

H. Wickham. ggplot2: Elegant Graphics for Data Analysis. Springer New York, 2009. URL http: //had.co.nz/ggplot2/book. [p151]

Paul Murrell

Department of Statistics

The University of Auckland

New Zealand

paul@stat.auckland.ac.nz 